\title{
Temporal and spatial variations of the magnetic field vector in sunspots
}

\author{
A. Settele ${ }^{1}$, M. Sigwarth ${ }^{2,3}$, and K. Muglach ${ }^{4}$ \\ 1 ESA Research and Scientific Support Department (RSSD), ESTEC, Keplerlaan 1, 2201 AZ, Noordwijk, The Netherlands \\ 2 National Solar Observatory/Sacramento Peak PO Box 62 Sunspot, NM 88349-0062, USA \\ ${ }^{3}$ Kiepenheuer-Institut für Sonnenphysik, Schöneckstr. 6, 79104 Freiburg, Germany \\ e-mail: msig@kis.uni-freiburg.de \\ 4 Astrophysikalisches Institut Potsdam, Telegrafenberg A27, 14473 Potsdam, Germany \\ e-mail: kmuglach@aip.de
}

Received 21 February 2002 / Accepted 28 May 2002

\begin{abstract}
In order to look for magnetic field vector oscillations in sunspots we used data measured with the Advanced Stokes Polarimeter at the Dunn Solar Telescope. We analyzed two time series of 65 and 110 min that were taken by scanning repeatedly a $6.24 \times 75^{\prime \prime}$ field of view, while obtaining the full Stokes vector in the lines FeI $630.15 \mathrm{~nm}$ and $630.25 \mathrm{~nm}$. An inversion was carried out and a Fast Fourier Transform (FFT) analysis was used to find oscillatory phenomena. We discuss possible unwanted effects that lead to apparent magnetic field oscillations and find an average amplitude of $(B, \psi) 5.8 \mathrm{G} / 0.23^{\circ}$ rms by excluding these effects, which also means that only $6 \%$ and $22 \%$ of the two sunspot umbrae respectively remained for analysis. If we smooth the power spectra over $2 \times 2$ pixels, all significant power disappears.
\end{abstract}

Key words. Sun: sunspots - Sun: magnetic fields - Sun: oscillations

\section{Introduction}

Sunspot oscillations in the form of velocity oscillations have been reported for more than 30 years (Beckers \& Talant 1969). It is well known that in sunspot umbrae and penumbrae the amplitude of the photospheric 5 min oscillations is reduced compared to the quiet sun (Balthasar et al. 1987; Lites 1992). At photospheric heights the 5 min oscillations are coherent over a significant fraction of the sunspot umbra (Penn \& LaBonte 1993; Bogdan 2000) and penumbra (Sigwarth \& Mattig 1997). The umbral 5 min oscillations are a response of the sunspot to the global solar mode or due to an internal excitation by magnetoconvection, whereas the 3 min oscillations are deduced as a resonance inside the sunspot (see Staude 1999; Bogdan 2000 for recent reviews). Such 3 min velocity oscillations are mainly observed above the umbra in the chromosphere (v. Uexküll et al. 1983; Thomas et al. 1984; Yoon et al. 1995). For the latter case there are two competing theories: First, a so-called fast-mode resonator (e.g. first paper: Uchida \& Sakurai 1975; recent paper: Lites et al. 1998) that is situated mostly below the solar surface between the increasing Alfvén speed in the photosphere and the overturning convection deeper inside the sunspot. And secondly a slow-mode resonator (e.g. first paper: Žugžda et al. 1983; recent paper: Settele et al. 2001), that is located between the rising temperature in the transition region

Send offprint requests to: A. Settele,

e-mail: asettele@rssd.esa.int and the photosphere. Both resonators may be realized in nature simultaneously (Thomas \& Weiss 1992).

A still controversial question is whether the magnetic field takes part in these oscillations, i.e. that the magnetic field vector $\boldsymbol{B}$ shows periodic variations as well. In spite of several observations (see Table 1) it is not in general accepted that observed oscillations of the field strength in the umbra and penumbra of sunspots are of solar origin. First Mogilevskij et al. (1972) reported umbral magnetic field strength $(B)$ oscillations in the 3 and 5 min period range in the central umbra of a sunspot near disk center. Milovanov (1980) found oscillations in two spots but none in three others. Gurman \& House (1981) detected periods of 3 and 5 min whereas Bachman (1983) found variations due to seeing influences. Thomas et al. (1984) didn't find any significant oscillations. In the 1990's Efremov \& Parfinenko (1996), Horn et al. (1997), Rüedi et al. (1998), Norton et al. (1998, 1999), Balthasar (1999) and Kupke et al. (2000) found oscillations in both 3 and 5 min, mainly at the umbral-penumbral border. Whereas Landgraf (1997) only found an enhanced but flat power spectrum in one point of the umbra and Lites et al. (1998) doubted their results as they consider the amplitude of the fluctuations as being too small to be a real signal: they found rms values of about $4 \mathrm{G}$ while others found oscillations up to $70 \mathrm{G}$ (see Table 1).

The theory for oscillations of the magnetic field strength is still being developed. Using the same MHD-model as for the velocity oscillations, where the sunspot as a whole 
Table 1. For each article the field of view (FOV) of the observation is shown; the method coded by (a) constant slit position, (b) spatial stepping with slit, (c) stepping through spectral line, (d) full spatial field, (m) magnetograph, (l) longitudinal $B$ field, (f) full $B$ field; the name of the instrument is given (magn. = magnetograph); the measured amplitude of magnetic field oscillations in Gauss: - no significant amplitude, ? existent but not given; region of observed oscillations and additional comments: rms the given amplitude is a rms value.

\begin{tabular}{|c|c|c|c|c|c|c|}
\hline article & FOV & method & instrument & $B[\mathrm{G}]$ & region & comments \\
\hline Mogilevskji et al. (1972) & $?$ & $(1-m)$ & IZMIRAN vec. magn. & $?$ & central umbra & \\
\hline Schultz et al. (1974) & $1 \mathrm{D}$ & (al-IV) & DST-Echelle spectr. & - & & $<36 \mathrm{G}$ noise level \\
\hline Milovanov (1980) & $?$ & $(1-m)$ & Kazakstan & $5-30$ & $?$ & \\
\hline Gurman et al. (1981) & $1 \mathrm{D}$ & (af-IQUV) & HAO-SP & $?$ & central umbra & \\
\hline Bachmann (1983) & $1 \mathrm{D}$ & (al-m) & magn. Einsteinturm & - & umbra & seeing induced \\
\hline Thomas et al. (1984) & $1 \mathrm{D}$ & (al) & DST-Echelle spectr. & - & & \\
\hline Efremov et al. (1996) & $1 \mathrm{D}$ & (al-m) & Pulkovo imag. magn. & $?$ & whole umbra & umbra in phase \\
\hline Horn et al. (1997) & $2 \mathrm{D}$ & (cd-IV) & FPI, VTT Tenerife & $-30^{*}$ & localized & ${ }^{*}$ from timeseries \\
\hline Landgraf (1997) & $1 \mathrm{D}$ & (b-IV) & GCT & - & & stray light \\
\hline Rüedi et al. (1998) & $2 \mathrm{D}$ & $(\mathrm{d}-\mathrm{m})$ & SOHO-MDI & 6.4 & localized & $\mathrm{rms}$ \\
\hline Lites et al. (1998) & $1 \mathrm{D}$ & (af-IQUV) & DST-ASP & - & umbra & $<4 \mathrm{G} \mathrm{rms}$ \\
\hline Norton et al. $(1998,1999)$ & $2 \mathrm{D}$ & (d-m) & SOHO-MDI & $?$ & & \\
\hline Balthasar (1999) & $2 \mathrm{D}$ & (cd-I) & FPI, VTT Tenerife & $40-70$ & pen-umbra border & rms \\
\hline Norton (2000) & $2 \mathrm{D}$ & (d-m) & SOHO-MDI & 18.1 & umbra & rms \\
\hline Kupke et al. (2000) & $2 \mathrm{D}$ & (bf-IQUV) & Mees spectrograph & 22 & pen-umbra border & $\mathrm{rms}$ \\
\hline Bellot Rubio et al. (2000) & $1 \mathrm{D}$ & (af-IQUV) & TIP, VTT Tenerife & - & & opacity \\
\hline Khomenko et al. (2001) & $1 \mathrm{D}$ & (af-IQUV) & TIP, VTT Tenerife & 10 & pen-umbra border & amplitude \\
\hline Norton et al. (2001) & $2 \mathrm{D}$ & (d-m) & SOHO-MDI & $?$ & umbra & phase analysis \\
\hline this paper & $2 \mathrm{D}$ & (bf-IQUV) & DST-ASP & -13.9 & localized & amplitude \\
\hline
\end{tabular}

without substructures is taken into account, Lites et al. (1998) point out that the expected rms of fluctuations in the magnetic field strength is about $0.5 \mathrm{G}$, which is an order of magnitude smaller than their observed fluctuations.

In order to explain field strength oscillations observed at the umbra-penumbra boundary, Zhugzhda et al. (2000) proposed the whispering gallery model. These whispering modes might not be excited in every sunspot and may exist only in parts of the umbra-penumbra border where given boundary conditions are fulfilled. The example in Zhugzhda et al. (2000) considers an ideal, circular, axis symmetric sunspot.

Other mechanisms are discussed (e.g. Norton et al. 1999; Bellot Rubio et al. 2000) that might produce measured oscillations in the magnetic field strength although $B$ itself does not oscillate: the acoustic waves running through the spot might shift the height of formation of the observed spectral lines which may lead to an apparent oscillation in field strength due to a field gradient in height. Or they may be simply an observational artifact produced by stray light coming from the quiet sun and scattered into the sunspot inside the Earth's atmosphere (Landgraf 1997).

Measurements of $\boldsymbol{B}$ oscillations are either based on data obtained with filter-spectrometers (2D field of view) or with slit-spectrographs (one spatial dimension). Most filterspectrometers are used for Stokes $I$ and Stokes $V$ measurements only. They cover a large field of view and offer good spatial resolution but the spectroscopic accuracy is typically much smaller than that of spectrograph-based polarimeters. Furthermore, there is cross-talk of the velocity signal into the magnetic field signal in the same order of magnitude as the assumed magnetic field oscillation (Settele et al. 2002). On the other hand, if the Stokes spectra are available only for a single slit position across the spot, spatial variations perpendicular to the slit can introduce artificial signals. To overcome this problem, in this paper we analyze time series of highprecision Stokes spectra for a small two-dimensional region across sunspots to investigate the spatial and temporal variation of the magnetic field vector $\boldsymbol{B}$. The combination of spatial coverage and Stokes vector analysis allows us to complement existing observations.

\section{Observations}

The observations were performed in June 2000 with the $\mathrm{HAO} / \mathrm{NSO}$ Advanced Stokes Polarimeter (ASP; Elmore et al. 1992; Lites 1996) at the NSO Dunn Solar Telescope, Sunspot, Sacramento Peak, New Mexico, USA. A spot tracker was used to reduce image motion. Time series were obtained by repeatedly scanning the spectrograph entrance slit in east-west direction across the sunspots. In order to achieve a temporal resolution of about 1 min, 12 steps per scan could be performed. With a step size of $00^{\prime \prime} .52$ and a pixel scale of $0 . .37$ pixel $^{-1}$ along the slit, the covered field of view was $66^{\prime \prime} 24 \times 75^{\prime \prime} 0$. The slit width corresponded to 0 ". 6 and the effective integration time per Stokes spectra was $2.1 \mathrm{~s}$. For the analysis we selected two time series of different spots. Figure 1 shows an example continuum data set and Table 2 presents the parameters for both datasets. Both spots were rather small, mature sunspots with well developed penumbrae. The spot AR 9034 observed on June 10 (hereafter SP1) included a lightbridge, whereas the umbra of AR 9036 observed on June 15 (hereafter SP2) was less disturbed. 


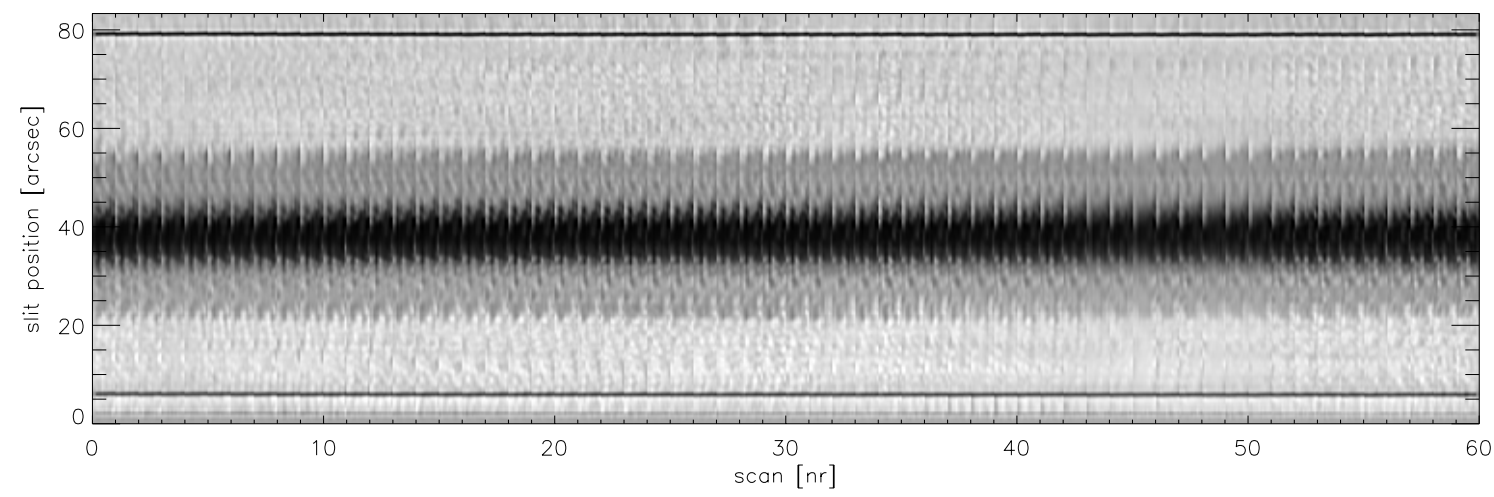

Fig. 1. Continuum time series of AR 9036, June 15, 2000, obtained with the ASP. The mean time between the individual scans is $60.86 \mathrm{~s}$ so that the whole shown series covers a little more than one hour. The two dark horizontal lines on the top and bottom of the image are used for calibration purposes and have its source from thin wires in front of the slit.

Table 2. Day of observation; active region number; position in solar latitude and longitude; distance from disc center; start time of observation (UT); number of scans; $\Delta t$ : mean time for one scan in seconds.

\begin{tabular}{lcllllc}
\hline \hline day & AR & position & $\cos \theta$ & time & scans & $\Delta t$ \\
\hline $06 / 10$ & 9034 & 10S 21E & 0.92 & $13: 24$ & 60 & 65.32 \\
$06 / 15$ & 9036 & 23S 08W & 0.90 & $13: 42$ & 109 & 60.86 \\
\hline
\end{tabular}

\section{Data calibration and analysis}

\subsection{Data reduction}

The Stokes spectra are calibrated according to the procedures described by Skumanich et al. (1997) including gain and crosstalk correction. The rms (root-mean square) noise in the continuum of Stokes $V, Q$, and $U$ is about $1 \times 10^{-3} I_{\mathrm{c}}$. Crosstalk between linear and circular polarization is corrected to about $1 \%$. Crosstalk from $I$ to $V, Q$, and $U$ is $<5 \times 10^{-3}$. Residual image motion between single scans is determined by shifting the individual continuum spectroheliograms in order to get maximum correlation. For the June 10 data a slight trend in the north-south $(y)$ direction is present which accounts for a maximum shift of 1.4 pixel or 0 "..5 over one hour ( 1 pixel or 0 "'.37 for the June 15 data). In the east-west direction $(x)$ the maximum shift is in between 0.2 and 0.3 pixel $\left(0{ }^{\prime \prime} .074\right.$ to $\left.00^{\prime \prime} 11\right)$. The eastwest shift was neglected in the correction as it is very small and it turned out not to change the time series significantly. In order to avoid the interpolation of the spectra, the examined $y$-shift vector is applied to the results of the Stokes inversion prior to the power analysis. The inversion of the full Stokes vector provides the magnetic field vector $\boldsymbol{B}$ for each data point with a polarization $P=\sqrt{Q^{2}+U^{2}+V^{2}} / I_{\mathrm{c}}>0.4 \%$. We applied the analytic "ASP inversion" to obtain the field strength $B$ and the field inclination $\psi$. The inversion is based on a Milne-Eddington atmosphere assuming one magnetic and one non-magnetic component (Skumanich \& Lites 1987). The line-of-sight velocity $v$ is determined from the FeI $630.15 \mathrm{~nm}$ line core shift relative to telluric $\mathrm{O}_{2}$ lines. By doing so wavelength drifts due to instrumental effects are compensated. Since we are only interested in the variation of the velocity, no absolute calibration for the velocities are performed.

\subsection{Time series analysis}

For all investigated parameters, the time series are interpolated linearly to equidistant time steps on basis of the average time between two measurements (Table 2) before the calculation of the power spectra. A forth order polynomial was subtracted from the time series to suppress trends in the data. Before calculating the power spectra, the first and the last $10 \%$ of the time series were apodised by a cosine bell. Unfortunately, this reduces the measured amplitudes a little bit, especially in short time series. To calculate the power spectra we use a Fast Fourier Transform (FFT). We defined three frequency bins: 2 to $4.5 \mathrm{mHz}$ ( $5 \mathrm{~min}$ ), 4.5 to $6.5 \mathrm{mHz}$ ( $3 \mathrm{~min}$ ) and $6.5 \mathrm{mHz}$ to Nyquist frequency (which we assume to be noise). This last bin is used to calculate the average noise level.

In order to get a reliable estimate of the significance of the peaks in the power spectra we carry out a number of different statistical tests: (i) First we calculate the average noise level, which is the mean value of the last frequency bin mentioned above. This is used to define the $99 \%$ significance level according to Groth (1975) for each pixel separately. (ii) The average of the power spectra of a $2 \times 2$ array of pixels is calculated to increase statistics and to suppress random peaks. Again the 99\% significance level is defined according to Groth (1975). (iii) Finally, we used a randomization test as outlined in Linnell Nemec \& Nemec (1985) and modified it according to Muglach (2002). This test compares the power spectrum with power spectra that were produced by randomly mixing the measured values of the sequence. This random mixing is repeated (here 1000 times). The fraction of times that a power peak of the original sequence is higher than the power peaks in the randomized ones gives the probability that the peak is due to an actual periodicity in the data. Note that this method looks for sinusoidal signals within noise. We are using a probability of $95 \%$ as a significance criterion. As we are not looking for lowfrequency oscillations in this study, we filter out all frequencies below $2 \mathrm{mHz}$ before starting the randomization. In addition, we apply the method iteratively as described in Muglach (2002).

Note that the two statistical tests (Groth and randomization) will only tell us if a measured power peak is due to a real periodicity in the data and not due to noise. One still has to consider 
if the power peaks that we find with the statistics are of solar origin or due to other effects outlined below.

\subsection{Possible misinterpretation}

The major problems in the interpretation of magnetic field oscillations are that they are expected to be small and that there are effects that can produce measured magnetic field oscillation although $\boldsymbol{B}$ itself does not oscillate. We will mention five of these effects in the following.

\section{- Instrumental effects}

One effect is due to instrumental cross-talk because of the method of measurement. When the complete spectral line is not taken simultaneously but by scanning through the spectral line with filters one gets cross-talk from the velocity signal into the magnetic signal. Settele et al. (2002) have studied this effect in detail. By using a spectrograph we avoid this effect.

The change of the dispersion of the spectrometer during the measurement was calculated from the two terrestrial $\mathrm{O}_{2}$ lines next to $\mathrm{Fe} \mathrm{I} 630.25 \mathrm{~nm}$. The dispersion of $11.99 \mathrm{m \AA} /$ pixel of June 10th (June 15th) fluctuates by about $0.07 \%(0.03 \%)$ which might lead to errors in the field strength of about $<2 \mathrm{G}(<1 \mathrm{G})$.

- Atmospheric effects/image motion

A third effect can happen due to image motion (e.g. seeing). To study the influence of image motion the following simulation was performed: as mentioned above (Sect. 3.1) we used the continuum maps to correct first order image motion. These shift vectors (in $x$ and $y$ ) were applied to the first map of the various parameters $(I, B, \psi, v)$, respectively. This means that an artificial time series was produced. Then the root mean square (rms) values in each pixel were calculated (see Tables 3 and 4). Without shifting the maps the rms of these time series would be zero as each map stays constant. In this way we simulate the residual image motion influence which will mainly be present in the regions of large gradients in the physical parameters. The magnetic field strength and the inclination will change simultaneously in such a region as the field is more vertical when the field strength is larger. Unfortunately, such a simultaneous change in $B$ and $\psi$ may also be valid for real $\boldsymbol{B}$ oscillations. The shift vectors measured from the data and used for the simulations represent image motion mainly caused by instrumental effects such as the spot tracker. Image motion due to seeing occurs also on smaller spatial and temporal scales and is therefore considered only to a small degree by the above simulation. The fourth effect is due to polarized and unpolarized stray light. In the latter case the magnetic field strength and the inclination will decrease when the continuum intensity is increasing as the Stokes vector is normalized to the continuum intensity. This effect should be reduced in our case as we use a two component model for the inversion (where the unpolarized component also contains unpolarized stray light), but it turns out that there are residual errors of this kind. In the case of polarized stray light the Zeeman splitting also might be influenced due to polarized light of regions with different field strength.

- Opacity effects

The fifth effect is connected to atmospheric waves that change the solar atmosphere's opacity. All sunspot models contain a gradient of field strength and temperature in height. Thus, if we look deeper into the sunspot photosphere the continuum intensity will increase as the temperature increases, the magnetic field will get stronger and the field vector becomes more vertical, and vice versa.

\section{Results and discussion}

\subsection{General overview and simulation}

Figures 2 and 3 show an overview of SP1 and SP2. The regions chosen for time series measurements are marked in Figs. 2a and $3 \mathrm{a}$ with the two vertical lines, and the direction to the center of the solar disk is given by the arrow. The mean values of their physical parameters $(I, B, \psi, v)$ and the absolute values of their derivatives in slit direction of the analyzed part of the spot are shown in Figs. $2 b$ and $3 b$. The analyzed region does not contain the quiet sun and is only including umbra and penumbra of the sunspots. The thin line marks the umbra-penumbra border in each plot. In SP1 the light bridge shows up clearly in the mean intensity plot of the time series (Fig. 2b). The rms of the time series (Figs. $2 b$ and $3 b$ ) exhibit large values in the regions of large spatial derivatives. Also the light bridge of SP1 can be slightly seen in the rms map of intensity. This reveals the limits of the first order reduction of image motion which is used in our case. Nevertheless, there are points of large rms where no large derivative appears. Tables 3 and 4 give an impression of the magnitude of the fluctuations in the observed sunspot umbrae. The average $B$ fluctuations are about $1 \%$ of the mean magnetic field strength. The image motion simulation shows that in principle it is possible that fluctuations of this order are produced by image motion effects: the maximum values in $B$, $\psi$, and $I$ are larger than the mean value of the real fluctuations of the measured time series. But the mean value of the simulated fluctuations is in most cases lower than the minimum value of the measured fluctuations of the time series, therefore it is very probable that we are able to find real oscillations. We further find that the velocity signal is barely influenced by image motion, as only regions with large gradients in the physical quantities are strongly disturbed by image motion, which is not the case for the velocity.

To avoid the misinterpretation because of uncorrected stray light the correlation of the negative magnetic field variation and the continuum intensity is plotted in the third last column of Figs. 2b and 3b. The Spearman correlation (Press et al. 1992) is used and only values of an absolute correlation larger than 0.5 are given. The strongest influence of stray light in SP1 seems to be near the light bridge and at the upper umbra-penumbra boundary which is marked by the thin solid line. Whereas in SP2 it is concentrated at both umbra-penumbra borders, it is not directly at the border in this case but slightly shifted towards the center of the sunspot where the umbra is already dark and shows no significant structure. 


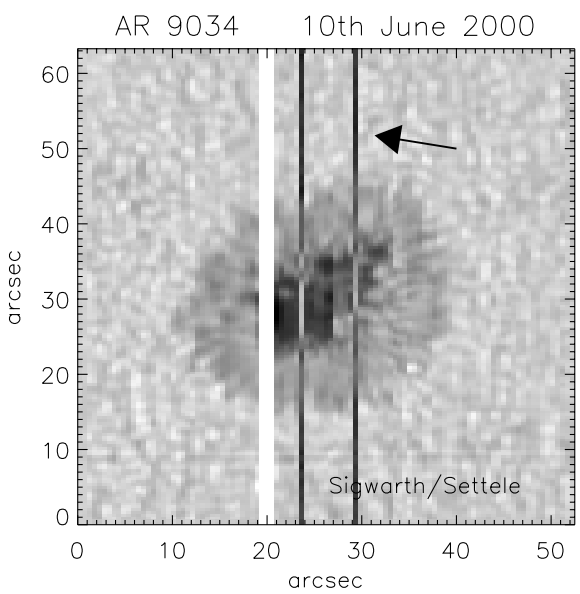

(a)

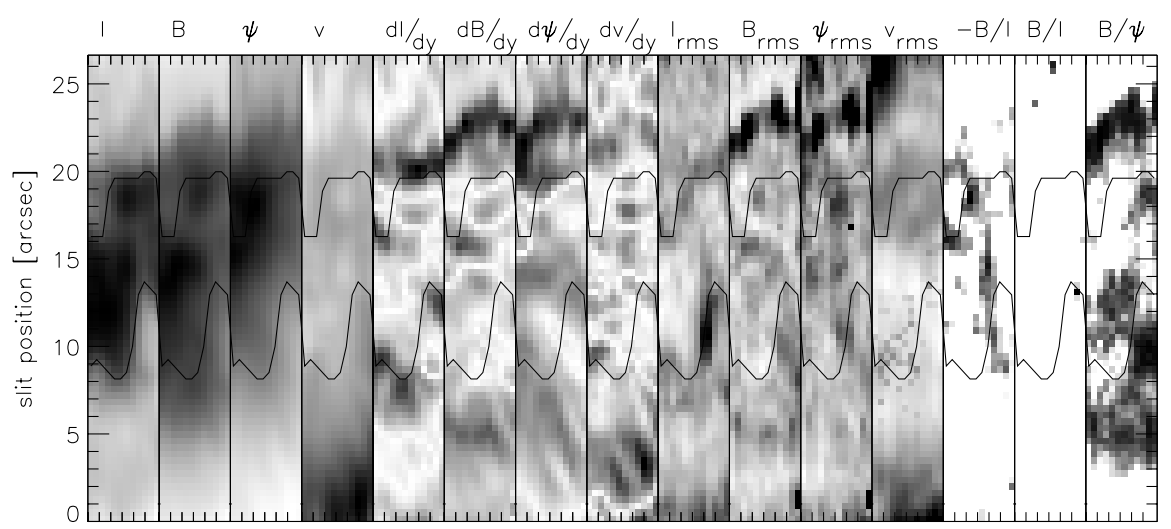

024024024024024024024024024024024024024024024 scan position [arcsec]

(b)

Fig. 2. a) Scan across the entire spot observed on June 10, 2000 (AR 9034), SP1. The vertical lines mark the region covered in the time series. The arrow points towards the disc center. b) Mean physical values and their absolute values of derivatives in the slit direction in the analyzed region of the continuum intensity $I$, magnetic field strength $B$, the field inclination $\psi$, and the velocity $v$. The values are averaged over the full time series. Then follow the root mean square and correlation maps. These maps show the rms values for $I, B$, $\psi$, and $v$ of the whole time series. The correlation show the negative/positive Spearman correlation of the magnetic field and intensity variation which are smaller/larger than $-/+0.5$, And the positive correlation of the magnetic field and the field inclination. In all columns except column one dark colours stand for large and light for small values. As the analyzed region contains only the umbra and part of the penumbra, the inner contour lines mark the umbra-penumbra boundary. Mean physical values of this region are given in Table 3.

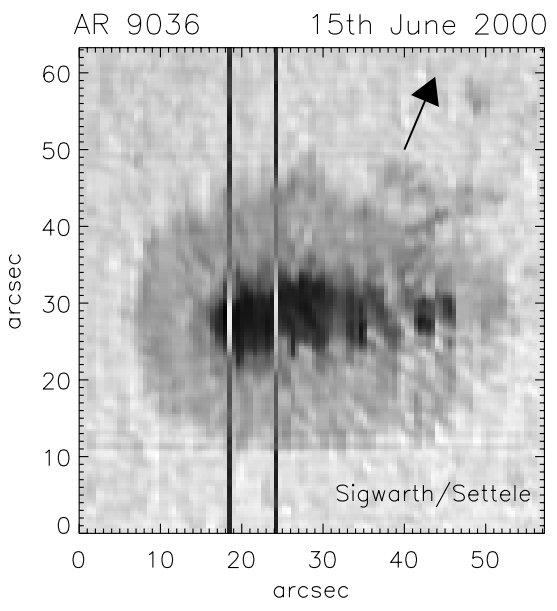

(a)

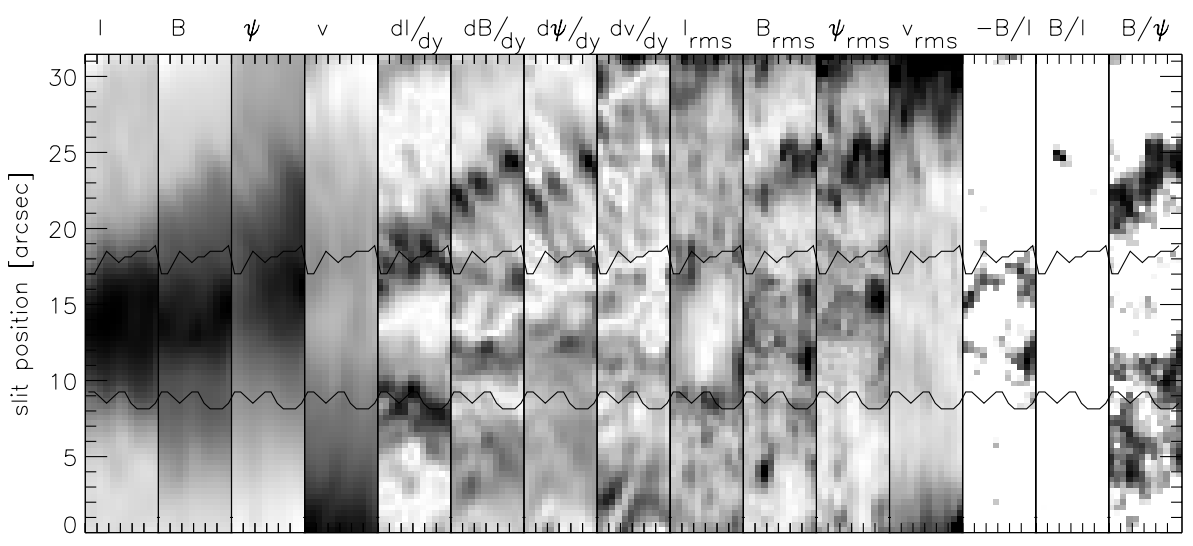

024024024024024024024024024024024024024024024 scan position $[\operatorname{arcsec}]$

(b)

Fig. 3. Similar to Fig. 2 but for the sunspot observed on June 15th, 2000 (AR 9036), SP2. a) Scan across the entire spot with the region marked used for time series scans. b) Mean physical values and their absolute values of derivatives in slit direction, rms values and Spearman correlation coefficients between continuum intensity/inclination and magnetic field. Mean physical values of this region are given in Table 4.

The same test was carried out for the other possibility: the opacity oscillations. In this case a positive correlation of larger than 0.5 between the magnetic field strength and the intensity was expected. But we found almost no positions that fulfill this condition as one can see from the second last column of Figs. $2 \mathrm{~b}$ and $3 \mathrm{~b}$. It is possible that this correlation is not very pronounced.

The changing of the opacity and image motion influences can be the reason for a large correlation between $B$ and $\psi$, especially in regions of large gradients (last column of Figs. $2 b$ and $3 b$ ).
Thus, in these regions plotted in the last three columns of Figs. $2 \mathrm{~b}$ and $3 \mathrm{~b}$, we are not able to differentiate between real $\boldsymbol{B}$ oscillations and those that can be produced by stray light or image motion. The map resulting by merging these three maps will be named "suppression map" in the following.

\subsection{The power maps}

In the literature, the locations where magnetic field oscillations are found and expected, the amplitude of the oscillations (see Table 1), whether real oscillations exist, or whether they are 
Table 3. The rms-values for the sunspot umbra SP1 of the time series of the dataset and the simulation (in brackets) are shown. The here analyzed region is located between the two thin solid lines in Fig. 2b. In the first row the maximum, minimum and mean value of the umbral magnetic field strength are given, which are the same for the simulation. Afterwards the fluctuations of the magnetic field strength, inclination and the velocities of the umbra and finally the velocities of the quiet sun are given.

\begin{tabular}{lccc}
\hline \hline & \multicolumn{3}{c}{ AR 9034 / 10th June (Simulation) } \\
\hline & Maximum & Minimum & Mean \\
\hline$B[\mathrm{G}]$ & 2503 & 1765 & 2103 \\
$B_{\mathrm{rms}}[\mathrm{G}]$ & 33.8 & 7.39 & 17.2 \\
& $(22.35)$ & $(1.52)$ & $(7.36)$ \\
$\psi_{\mathrm{rms}}\left[{ }^{\circ}\right]$ & 2.06 & 0.47 & 0.95 \\
& $(1.32)$ & $(0.02)$ & $(0.53)$ \\
$I_{\mathrm{rms}}[$ Counts $]$ & 217.3 & 19.5 & 79.1 \\
& $(111.2)$ & $(3.14)$ & $(30.2)$ \\
$v_{\mathrm{rms}}\left[\frac{\mathrm{m}}{\mathrm{s}}\right]$ & 144.0 & 73.9 & 112.5 \\
& $(110.6)$ & $(0.53)$ & $(9.12)$ \\
$v_{\text {rms }}\left[\frac{\mathrm{m}}{\mathrm{s}}\right]$ & 467.7 & 211.9 & 323.0 \\
$\quad$ (quiet sun) & $(125.6)$ & $(2.34)$ & $(37.8)$ \\
\hline
\end{tabular}

Table 4. The rms-values for the sunspot umbra SP2 of the time series of the dataset and the simulation (in brackets) are shown. The here analyzed region is located between the two thin solid lines in Fig. $3 \mathrm{~b}$. For more information see Table 3.

\begin{tabular}{lccc}
\hline \hline & \multicolumn{3}{c}{ AR 9036 / 15th June (Simulation) } \\
\hline & Maximum & Minimum & Mean \\
\hline$B[\mathrm{G}]$ & 2815 & 1949 & 2386 \\
$B_{\mathrm{rms}}[\mathrm{G}]$ & 55.4 & 11.8 & 25.9 \\
& $(24.4)$ & $(0.87)$ & $(7.8)$ \\
$\psi_{\text {rms }}\left[{ }^{\circ}\right]$ & 2.87 & 0.48 & 1.34 \\
& $(1.27)$ & $(0.04)$ & $(0.49)$ \\
$I_{\text {rms }}[$ Counts $]$ & 167.9 & 30.6 & 81.2 \\
& $(82.5)$ & $(0.64)$ & $(23.3)$ \\
$v_{\text {rms }}\left[\frac{\mathrm{m}}{\mathrm{s}}\right]$ & 140.4 & 64.7 & 89.0 \\
& $(33.0)$ & $(0.48)$ & $(10.6)$ \\
$v_{\text {rms }}\left[\frac{\mathrm{m}}{\mathrm{s}}\right]$ & 324.6 & 154.9 & 221.8 \\
(quiet sun) & $(84.9)$ & $(1.1)$ & $(23.7)$ \\
\hline
\end{tabular}

introduced by the effects mentioned in Sect. 3.3 are controversial points. To find the locations, two dimensional power maps are commonly presented. But there are different ways of producing them. Therefore, we show five different possibilities to make power maps in Fig. 5 for both sunspots for comparison:

(a) we summed all power in the each pixel that was confirmed by a $95 \%$ probability limit using the randomization test in each pixel, (b) the mean of the power spectra of a $2 \times 2$ array of pixels is taken to increase statistics and to suppress random peaks. The $99 \%$ Groth-level was subtracted from the maximum power peak in the frequency region and only the remaining positive power was plotted. (c) the same as (b) without smoothing, (d) the mean power of every frequency bin is plotted with subtraction of the average noise, and finally (e) the same as (d) but no subtraction of noise.

Starting from the bottom image, Fig. 5e, of both days, we see that when we just take the mean of the frequency regions (2-4.5 $\mathrm{mHz}$ for $5 \mathrm{~min}$ and $4.5-6.5 \mathrm{mHz}$ for $3 \mathrm{~min}$ oscillations) we will get mainly enhanced power in the regions of large gradients which we have already seen in Figs. 2 and 3. Even if we subtract the noise (mean power of $6.5 \mathrm{mHz}-\mathrm{Nyquist}$ frequency) as is shown in Fig. 5d the main features of large power at the large gradients still exists. This method seems not to be appropriate for terrestrial observations of the magnetic field and intensity if we are only able to correct the first order of image motion, except if we are just interested in velocity, as their power maps look very similar in the Figs. 5a-e. Furthermore, the power maps in Fig 5e are sensitive to isolated outliers in the time series, as can be seen e.g. at the dark pixel in the umbra of the inclination plots of SP1 (Fig. 5e, slit position 4".2, scan position $16^{\prime \prime} .7$ ). At one time step of this series the inclination and magnetic field differs very strongly from the other values, respectively. By nature, this leads to heightened power in all frequencies and the pixel nearly disappears if we subtract our defined noise in Fig. 5d.

When we use the Groth-level to discriminate all insignificant periodic signals in Fig. 5c, we see that most of the large signals in the region of large gradients disappear. But there is one feature in SP2 where we do not see a large gradient but which appears in the rms plot of the magnetic field Fig. $3 \mathrm{~b}$ and in the mean power maps (Figs. 5d-e): At scan position 2.'0 and slit position 4 "'0. This small part belongs to a sudden increase of the magnetic field strength and decrease in the intensity which then moves towards the umbra. Of course this can only be seen in a movie sequence and has nothing to do with the oscillations we look for. But we should keep in mind that if we only had a fixed slit, we would not have identified this object. This (moving magnetic feature) might lead to misinterpretations in cases of fixed slit observations.

Coming back to the oscillations and increasing the confidence by increasing the statistics we summed the power spectra of 4 adjacent pixels (Fig. 5b). We see that most of the small hints of periodic oscillations vanish. This method may be too rigorous but it shows how marginal these oscillations are. On the other hand this can also mean that the $\boldsymbol{B}$ oscillations happen on a very small spatial scale.

Considering the last and most time consuming method to look for significant peaks we plotted the power maps confirmed by randomization on the top Fig. 5a. The comparison with the Groth-level methods shows that the results are similar but each method has its region where it is more draconic than the other method. This last method also finds oscillations in the region of the large gradients which seems to be a little more suppressed by the Groth-level, on the other hand it eliminates nearly all 3 min velocity oscillations inside the umbra.

In the following we will use Figs. 5a-c for further discussion only.

\subsubsection{Velocity oscillation}

The 5 min velocity oscillations are present in the umbra and penumbra, and the 3 min oscillations are mainly concentrated inside the umbra. This is more pronounced in SP1 than in SP2. The rms of the time series in the umbra are reduced by a factor of 2.5-2.9 compared to the quiet sun (Tables 3 and 4). 


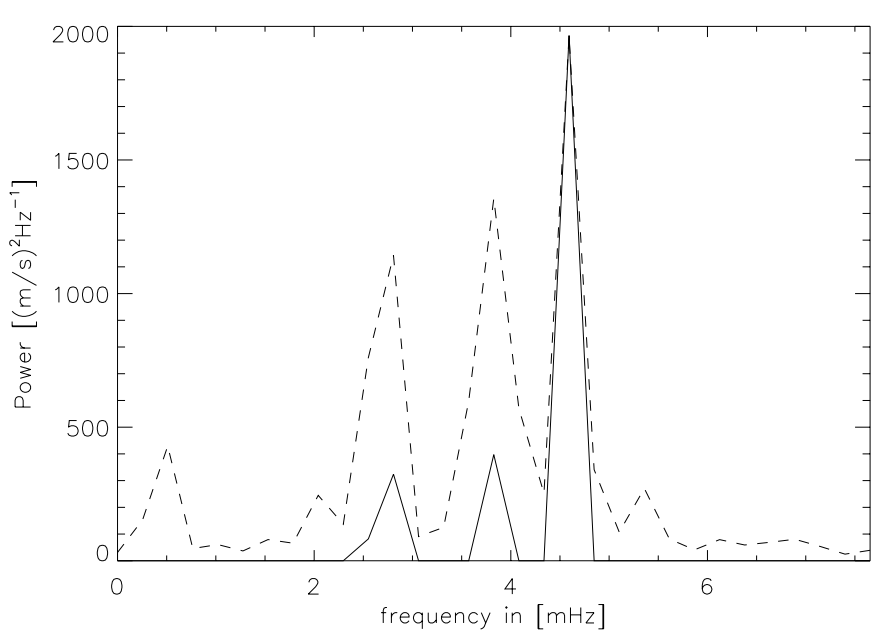

Fig. 4. Mean velocity power spectra of SP1 at region around position $\left(2{ }^{\prime \prime} 5,11^{\prime \prime} 5\right)$ in the umbra. The solid lines belong to power confirmed by the randomization test. The dashed lines to normal power spectrum

As stated before, the randomization method suppresses nearly all $3 \mathrm{~min}$ velocity oscillations inside the umbra. However, one nearly circular region of enhanced 3 min velocity power is still there in SP1 at scan position 2".5 and slit position $11^{\prime \prime} 5$. A mean velocity power spectrum of this area is given in Fig. 4. This may be interpreted as the signature of the lower part of a slow-mode resonator (see Žugžda et al. 1983; Settele et al. 2001 and references therein). The slow-mode resonator is mainly an acoustic resonator that is guided by the magnetic field lines. As it needs two reflection boundaries (the lower photosphere and the transition region) a direct connection between these regions is needed. This is not the case in the penumbra as the magnetic field lines become horizontal and do not reach the transition region in the required distance and therefore no $3 \mathrm{~min}$ velocity oscillations can be found in the penumbra. It is very seldom that 3 min oscillations are seen in FeI $630.15 \mathrm{~nm}$ lines in the umbra as the amplitude is expected to be very small in the photosphere. Unfortunately, we have no simultaneous measurements of velocities in higher atmospheric levels to support our assumption.

\subsubsection{Magnetic field oscillation}

When we take a mean power spectrum of each sunspot umbra respectively, we do not find any significant power in the magnetic field strength and inclination.

At first glance it looks like we have found some localized magnetic field oscillations in the umbra, as in Figs. 5a-c we see power indicated in the maps. Unfortunately, most of it disappears in Fig. 5b. Without knowing whether they are real or not, it first can be noted that there seem to be no preferred region of appearance, as found by other authors (Table 1).

Furthermore, we excluded also oscillations which may be artifacts of the observation by the "suppression map". Only $22.6 \%$ of SP1 and $34.9 \%$ of SP2 remain for analysis. The new power maps are shown in Figs. 6 and 7 for SP1 and SP2, respectively. Again, here is no evidence for a preferred region
Table 5. SP1 umbra - The maximum and the mean $B$ and $\psi$ amplitudes in the $3 \mathrm{~min}$ and $5 \mathrm{~min}$ frequency bands are given. The specific pixels have to fulfill the Groth or Randomization criteria. Additionally the "suppression map" (map) is required, which excludes the strong correlation of the last three columns of Fig. 2b. The same analysis done without "suppression map" is marked by "no map". The analyzed umbra consists of 288 pixel (the whole plotted region has 864 pixel), after suppression of possible unwanted areas (map) 65 umbral pixel remain, $n$ gives the number of pixels which fulfill the conditions, this also given in percentage of the whole umbra. The $x, y$-position in [arcsec] of the maximum is given.

\begin{tabular}{|c|c|c|c|c|c|}
\hline $3 \mathrm{~min}$ & $\max$ & mean & $n$ & $\%$ & position \\
\hline \multirow[t]{3}{*}{ Groth } & $10.7 \mathrm{G}$ & $6.8 \mathrm{G}$ & 6 & 2.1 & $(2.60,15.17)$ \\
\hline & $0.27^{\circ}$ & $0.27^{\circ}$ & 2 & 0.7 & $(4.16,19.61)$ \\
\hline & $12.3 \mathrm{G}$ & $7.4 \mathrm{G}$ & 33 & 11.5 & $(0.52,15.54)$ \\
\hline & $0.59^{\circ}$ & $0.32^{\circ}$ & 18 & 6.3 & $(3.12,17.76)$ \\
\hline \multirow[t]{4}{*}{ Randon } & $7.6 \mathrm{G}$ & $7.6 \mathrm{G}$ & 1 & 0.3 & $(5.20,15.91)$ \\
\hline & - & - & - & - & - \\
\hline & $11.1 \mathrm{G}$ & $9.2 \mathrm{G}$ & 5 & 1.7 & $(3.64,19.61)$ \\
\hline & $0.59^{\circ}$ & $0.50^{\circ}$ & 3 & 1.0 & $(3.12,17.76)$ \\
\hline $5 \mathrm{~min}$ & $\max$ & mean & $n$ & $\%$ & position \\
\hline \multirow[t]{4}{*}{ Groth } & $13.1 \mathrm{G}$ & $8.8 \mathrm{G}$ & 16 & 5.6 & $(2.60,15.17)$ \\
\hline & $0.55^{\circ}$ & $0.37^{\circ}$ & 15 & 5.2 & $(0.52,13.69)$ \\
\hline & $16.7 \mathrm{G}$ & $9.0 \mathrm{G}$ & 69 & 24.0 & $(2.08,15.54)$ \\
\hline & $0.55^{\circ}$ & $0.35^{\circ}$ & 47 & 16.3 & $(0.52,13.69)$ \\
\hline \multirow[t]{2}{*}{ Random map } & $13.9 \mathrm{G}$ & $9.8 \mathrm{G}$ & 12 & 4.2 & $(2.08,15.17)$ \\
\hline & $0.55^{\circ}$ & $0.42^{\circ}$ & 9 & 3.1 & $(0.52,13.69)$ \\
\hline \multirow[t]{2}{*}{ no map } & $16.7 \mathrm{G}$ & $9.7 \mathrm{G}$ & 51 & 17.7 & $(2.08,15.54)$ \\
\hline & $0.55^{\circ}$ & $0.38^{\circ}$ & 34 & 11.8 & $(0.52,13.69)$ \\
\hline
\end{tabular}

for oscillations. Tables 5 and 6 give an overview of the maximum and mean amplitudes of the magnetic field oscillations with the two significance criteria, with and without the use of the "suppression maps" to eliminate unwanted power. The number of pixels, that remain after the suppression and show significant power, is given. We see that less than $6 \%$ of the umbra in SP1 and less than $22 \%$ of the SP2 umbra show significant magnetic field oscillations. The maximum amplitude value of $25.6 \mathrm{G} / 0.87^{\circ}(B, \psi)$ in SP2 without the "suppression map" is clearly due to stray light (cf. Fig. 3b). If we suppress all discussed possible artifacts, we find a mean amplitude value of the magnetic field oscillation of both sunspots of about $8.2 \mathrm{G} / 0.32^{\circ}\left(5.8 \mathrm{G} / 0.23^{\circ} \mathrm{rms}\right)$ and a maximum value of $13.9 \mathrm{G} / 0.74^{\circ}\left(9.8 \mathrm{G} / 0.52^{\circ} \mathrm{rms}\right)$. This is somewhat at the lower end of what was found before (see Table 1). In comparison to Lites et al. (1998) who used the same instrument but with a spatially fixed slit, a much shorter cadence and no artifact suppression, our mean amplitude is slightly larger than their upper limit of $4 \mathrm{G} / 0.09^{\circ} \mathrm{rms}$ which they considered to be an instrumental effect and inversion cross-talk from the velocity. Nevertheless, it might be that our conditions for exclusion were too restrictive, as it is possible that there are real magnetic field oscillations were a measurable change in inclination is parallel to the change in the field strength which we assigned mainly to image motion effects. 

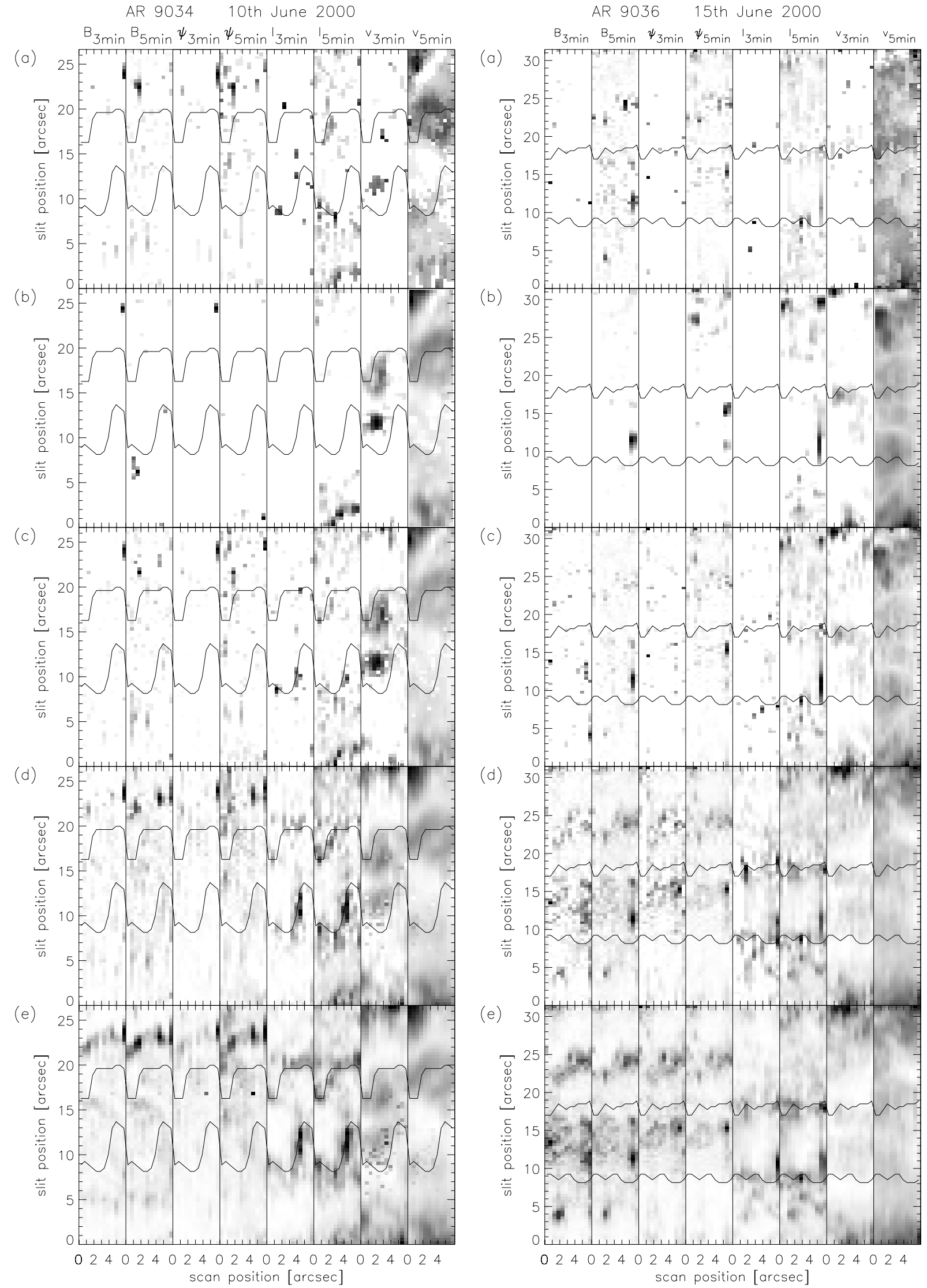

Fig. 5. Power maps of the umbra and penumbra of SP1 and SP2. a) Power confirmed by randomization, b) mean spectra over 4 pixel and power confirmed by Groth-Level, c) power confirmed by Groth-Level, d) mean power in frequency region, noise subtracted, but no significance test of power $\mathbf{e}$ ) mean power in frequency region. The darker the regions the larger power is present and white means that no significant power was found. 
Table 6. The same as Table 5, but for SP2 umbra. The corresponding map compounds of the last three columns of Fig. 3b. The analyzed umbra consists of 315 pixel (the whole plotted region has 1020 pixel), after suppression of possible unwanted areas (map) 110 umbral pixel remain.

\begin{tabular}{|c|c|c|c|c|c|}
\hline $3 \mathrm{~min}$ & $\max$ & mean & $n$ & $\%$ & position \\
\hline \multirow[t]{3}{*}{ Groth } & $12.9 \mathrm{G}$ & $6.9 \mathrm{G}$ & 16 & 5.1 & $(0.52,13.69)$ \\
\hline & $0.28^{\circ}$ & $0.22^{\circ}$ & 15 & 4.8 & $(4.68,14.43)$ \\
\hline & $13.4 \mathrm{G}$ & $7.4 \mathrm{G}$ & 40 & 12.7 & $(5.72,11.10)$ \\
\hline & $0.39^{\circ}$ & $0.22^{\circ}$ & 40 & 12.7 & $(1.04,14.43)$ \\
\hline \multirow[t]{4}{*}{ Random } & $12.9 \mathrm{G}$ & $9.9 \mathrm{G}$ & 2 & 0.6 & $(0.52,13.69)$ \\
\hline & $0.27^{\circ}$ & $0.27^{\circ}$ & 1 & 0.3 & $(4.68,12.58)$ \\
\hline & $13.4 \mathrm{G}$ & $10.4 \mathrm{G}$ & 4 & 1.3 & $(5.72,11.10)$ \\
\hline & $0.39^{\circ}$ & $0.29^{\circ}$ & 5 & 1.6 & $(1.04,14.43)$ \\
\hline $5 \mathrm{~min}$ & $\max$ & mean & $n$ & $\%$ & position \\
\hline \multirow[t]{4}{*}{ Groth } & $13.4 \mathrm{G}$ & $8.4 \mathrm{G}$ & 59 & 18.7 & $(0.52,13.32)$ \\
\hline & $0.74^{\circ}$ & $0.30^{\circ}$ & 68 & 21.6 & $(5.20,14.80)$ \\
\hline & $25.6 \mathrm{G}$ & $10.4 \mathrm{G}$ & 167 & 53.0 & $(5.20,11.47)$ \\
\hline & $0.87^{\circ}$ & $0.30^{\circ}$ & 173 & 54.9 & $(5.20,15.17)$ \\
\hline \multirow[t]{4}{*}{ Random } & $12.0 \mathrm{G}$ & $8.2 \mathrm{G}$ & 30 & 9.5 & $(2.08,13.32)$ \\
\hline & $0.74^{\circ}$ & $0.34^{\circ}$ & 37 & 11.8 & $(5.20,14.80)$ \\
\hline & $25.6 \mathrm{G}$ & $11.0 \mathrm{G}$ & 114 & 36.2 & $(5.20,11.47)$ \\
\hline & $0.87^{\circ}$ & $0.33^{\circ}$ & 100 & 31.7 & $(5.20,15.17)$ \\
\hline
\end{tabular}

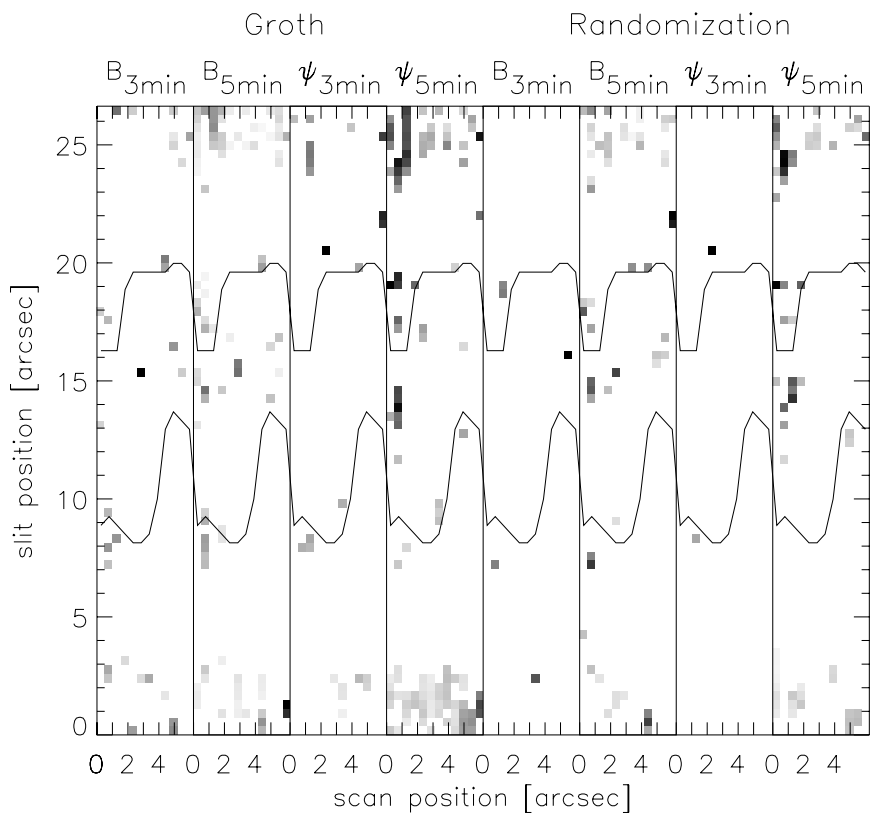

Fig. 6. Power maps of the umbra/penumbra SP1 which fulfill the Groth resp. the Randomization criteria and exclude the regions of larger correlations of the last three columns in Fig. 2b by the "suppression map".

\section{Conclusions and outlook}

Looking for magnetic field oscillations is like looking for a needle in a haystack. A lot of observational artifacts introduce oscillations in the measures of the magnetic field vector and have to be separated from real physical oscillations of solar origin. But, with our strong selection process, which excludes stray light, image motion and opacity effects, we find an average rms $(B, \psi)$ amplitude of $5.8 \mathrm{G} / 0.23^{\circ}$ (including 3 and 5 min

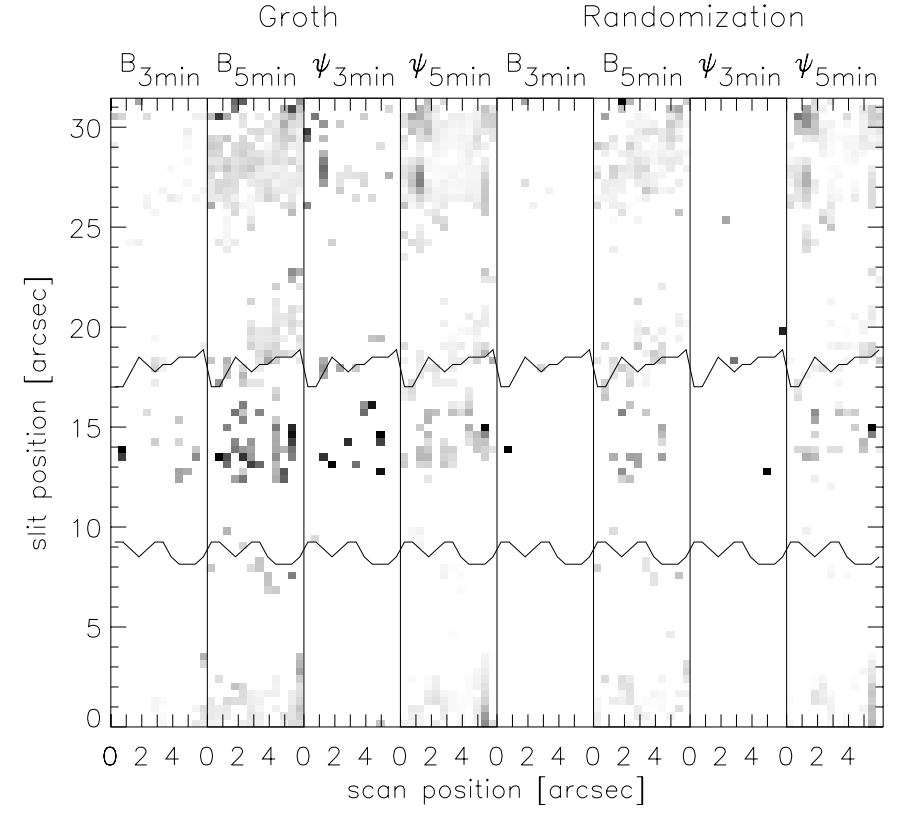

Fig. 7. Power maps of the umbra/penumbra SP2 which fulfill the Groth resp. the Randomization criteria and exclude the regions of larger correlations of the last three columns in Fig. 3b by the "suppression map".

oscillations) in the two analyzed sunspot umbrae and a maximum rms amplitude of $9.8 \mathrm{G} / 0.52^{\circ}$. A preferred location of appearance for the oscillations inside the sunspot umbra was not found. To make further progress in this research area we need to reduce the effect of image motion either by using adaptive optics on the ground, or by doing spectropolarimetry from space, e.g. Solar-B (Lites et al. 2001) or Sunrise (Schmidt et al. 2001). Increasing Zeeman sensitivity and reducing stray light effects by using infrared lines will also help, especially in combination with larger telescopes like GREGOR (v.d. Lühe et al. 2001) or the ATST (Keil et al. 2001) to get the necessary spatial resolution. Another question for future studies, which was not addressed here, will be the propagation of the oscillations into the higher layers of the sunspot atmosphere, to verify our observation of the slow-mode resonator.

Acknowledgements. The observations and main parts of the data reduction were done during a 3 month stay of AS as Summer Research Assistant at Sunspot, Sacramento Peak, NM, USA. It was financed by NSO via AURA. A part of this work was supported by the German Science Foundation (DFG) through the grant STA 351/5-3. This research is part of the European Solar Magnetometry Network (ESMN) supported by the EC through the TMR programme and used facilities of the ESA and AIP in this context.

\section{References}

Bachmann, G. 1983, Phys. Solariterr. Potsdam, 20, 29

Balthasar, H. 1999, Sol. Phys., 187, 389

Balthasar, H., Küveler, G., \& Wiehr, E. 1987, Sol. Phys., 112, 37

Beckers, J. M., \& Talant, P. E. 1969, Sol. Phys., 7, 351

Bellot Rubio, L. R., Collados, M., Ruiz Cobo, B., \& Rodrigguez Hidalgo, I. 2000, ApJ, 534, 989

Bogdan, T. J. 2000, Sol. Phys., 192, 373

Elmore, D. F., Lites, B. W., Tomczyk, S., et al. 1992, Proc. SPIE, 1746, 22 
Efremov, V. I., \& Parfinenko, L. D. 1996, Astron. Rep., 40, 89

Gurman, J. B., \& House, L. L. 1981, Sol. Phys., 71, 5

Groth, E. 1975, ApJS, 29, 285

Horn, T., Staude, J., \& Landgraf, V. 1997, Sol. Phys., 172, 69

Keil, S. L., Rimmele, T. R., Keller, C. U., et al. 2001, in Advanced Solar Polarimetry-Theory, Observation, and Instrumentation, ed. M. Sigwarth, ASP Conf. Ser., 236, 597

Kupke, R., Labonte, B. J., \& Mickey, D. L. 2000, Sol. Phys., 191/1, 97

Landgraf, V. 1997, Astron. Nachr., 318, 129

Linnell Nemec, A. F., \& Nemec, J. M. 1985, AJ, 90, 2317

Lites, B. W. 1992, in Sunspots: Theory and Observations, ed. J. H. Thomas, \& N. O. Weiss (Kluver), NATO-ASI Ser., 375, 261

Lites B. W. 1996, Sol. Phys., 163, 223

Lites, B. W., Thomas, J. H., Bogdan, T. J., \& Cally, P. S. 1998, ApJ, 497, 464

Lites, B. W., Elmore, D. F., \& Streander, K. V. 2001, in Advanced Solar Polarimetry-Theory, Observation, and Instrumentation, ed. M. Sigwarth, ASP Conf. Ser., 236, 33

v.d.Lühe, O., Schmidt, W., Soltau, D., Berkefeld, T., Kneer, F., \& Staude, J. 2001, Astron. Nachr., 322, 353

Mogilevskij, E. F., Obridko, V. N., \& Shelting, B. D. 1972, Astron. Tsirk. SSR, 669, 1

Milovanov, V. N. 1980, Phys. Solariterr. Potsdam, 13, 109

Muglach, K. 2002, A\&A, in preparation

Norton, A. A., Ulrich, R. K., Bogart, R. S., Bush, R. I., \& Hoeksema, J. T. 1998, IAU-Symp., 185, 453

Norton, A. A., Ulrich, R. K., Bush, R. I., \& Tarbell, T. D. 1999, ApJ, 518, L123

Norton, A. A. 2000, Univerity of California, Los Angeles, Ph.D. Dissertation, Study of Solar Photospheric MHD Oscillations
Norton, A. A., \& Ulrich, R. K. 2001, ApJ, 561, 435

Penn, M. J., \& LaBonte, B. J. 1993, ApJ, 415, 383

Press, W. H., Teukolsky, S. A., Vetterling, W. T., \& Flannery, B. P. 1992, Numerical Recipes, The Art of Scientific Computing, second edition (Cambridge University Press), sec. 14.6

Rüedi, I., Solanki, S. K., Stenfo, J. O., Tarbell, T., \& Scherrer, P. H. 1998, A\&A, 335, L97

Schmidt, W., Solanki, S. K., Lites, B. W., Title, A., \& Martinez Pillet, V. 2001, Astron. Nachr., 322, 363

Schultz, R. B., \& White, O. R. 1974, Sol. Phys., 35, 309

Settele, A., Staude, J., \& Zhugzhda, Y. D. 2001, Sol. Phys., 202, 281

Settele, A., Carroll, T. A., Nickelt, I., \& Norton, A. A. 2002, A\&A, 386,1123

Sigwarth, M., \& Mattig, W. 1997, A\&A, 324, 743

Skumanich, A., \& Lites, B. W. 1987, ApJ, 322, 473

Skumanich, A., Lites, B. W., Martínez Pillet, V., et al. 1997, ApJS, 110,357

Staude, J. 1999, in Third Advances in Solar Physics Euroconference: Magnetic Fields and Oscillations, ed. B. Schmieder, A. Hofmann, \& J. Staude, ASP Conf. Ser., 184, 113

Thomas, J. H., Cram, L. E., \& Nye, A. H. 1984, ApJ, 285, 368

Thomas, J. H., \& Weiss, O. W. 1992, NATO ASI Ser. C, 375, 3

Uchida, Y., \& Sakurai, T. 1975, Publ. Astron. Soc. Japan, 27, 259

v. Uexküll, M., Kneer, F., \& Mattig, W., A\&A, 123, 263

Yoon, T.-S., Yun, H.-S., Kim, J.-H. 1995, J. of the Korean Astron. Soc., 28, 245

Žugžda, Y. D., Locāns, V., \& Staude, J. 1983, Sol. Phys., 82, 369

Zhugzhda, Y. D., Balthasar, H., \& Staude, J. 2000, A\&A, 355, 347 